\title{
Differential timing of neurogenesis underlies dorsal-ventral topographic projection of olfactory sensory neurons
}

\author{
Eerdunfu $^{1 \dagger}$, Naoki Ihara ${ }^{2+}$, Bao Ligao ${ }^{2}$, Yuji Ikegaya ${ }^{2,3}$ and Haruki Takeuchi ${ }^{2,4^{*}}$ (D)
}

\begin{abstract}
Background: The mammalian primary olfactory system has a spatially-ordered projection in which olfactory sensory neurons (OSNs) located in the dorsomedial (DM) and ventrolateral $(V L)$ region of the olfactory epithelium (OE) send their axons to the dorsal and ventral region of the olfactory bulb $(\mathrm{OB})$, respectively. We previously found that OSN axonal projections occur sequentially, from the DM to the VL region of the OE. The differential timing of axonal projections is important for olfactory map formation because early-arriving OSN axons secrete guidance cues at the OB to help navigate late-arriving OSN axons. We hypothesized that the differential timing of axonal projections is regulated by the timing of OSN neurogenesis. To test this idea, we investigated spatiotemporal patterns of OSN neurogenesis during olfactory development.

Methods and results: To determine the time of OSN origin, we used two thymidine analogs, BrdU and EdU, which can be incorporated into cells in the S-phase of the cell-cycle. We injected these two analogs at different developmental time points and analyzed distribution patterns of labeled OSNs. We found that OSNs with different dates of origin were differentially distributed in the OE. The majority of OSNs generated at the early stage of development were located in the DM region of the $O E$, whereas OSNs generated at the later stage of development were preferentially located in the $V L$ region of the OE.

Conclusions: These results indicate that the number of OSNs is sequentially increased from the DM to the VL axis of the OE. Moreover, the temporal sequence of OSN proliferation correlates with that of axonal extension and emergence of glomerular structures in the OB. Thus, we propose that the timing of OSN neurogenesis regulates that of OSN axonal projection and thereby helps preserve the topographic order of the olfactory glomerular map along the dorsal-ventral axis of the $\mathrm{OB}$.
\end{abstract}

Keywords: Olfactory receptor, Olfactory sensory neuron, Neural circuit formation, Topographic map, Zonal organization, Neurogenesis

\section{Background}

In the mouse olfactory system, olfactory receptor (OR) genes form a multigene family comprising $>1000$ genes [1]. Of this rich repertoire of genes, an individual olfactory sensory neuron (OSN) expresses only one functional OR [2]. OSN axons that express a given type of OR converge

\footnotetext{
* Correspondence: haruki-t@mol.f.u-tokyo.ac.jp

${ }^{\dagger}$ Equal contributors

${ }^{2}$ Laboratory of Chemical Pharmacology, Graduate School of Pharmaceutical

Sciences, the University of Tokyo, Tokyo 113-0033, Japan

${ }^{4} J a p a n$ Science and Technology Agency (JST), PRESTO, 4-1-8 Honcho

Kawaguchi, Saitama 332-0012, Japan

Full list of author information is available at the end of the article
}

to a few spatially invariant glomeruli in the olfactory bulb (OB) [3-5], generating an olfactory topographic map. The development of the olfactory topographic map comprises initial global targeting and subsequent activity-dependent refinement $[6,7]$. The global targeting process is genetically determined and regulated by two independent mechanisms that control different axes of axonal extension in the OB. Axonal projections along the anterior-posterior axis are dependent on the expressed OR species [8]. Several axon-guidance molecules, such as Neuropilin-1 and Plexin-A1, whose expression levels are regulated by expressed OR molecules have been proposed to participate in anterior-posterior targeting [9-11]. In contrast, as 
for the dorsal-ventral (D-V) targeting of OSN axons, there is a close correlation between OSN positions in the olfactory epithelium $(\mathrm{OE})$ and glomerular locations in the $\mathrm{OB}$ [12]. The OE can be roughly divided into two domains on the basis of genetic markers: dorsal (D)-zone and ventral (V)-zone. D-zone OSNs, which are located in the dorsomedial region of the $\mathrm{OE}$, send their axons to the dorsal region of the OB, whereas V-zone OSNs, which are located in the ventrolateral region of the $\mathrm{OE}$, project their axons to the ventral region of the OB. The expression patterns of OR genes are different between the D- and Vzones. In the D-zone, the expression patterns of OR genes are randomly distributed throughout the OE [13]. However, V-zone specific OR genes show spatially limited expression. Each OR gene possesses a unique expression domain and these domains are arranged in a continuous and overlapping manner within the V-zone [14-16]. Several studies have shown that the topographic order of glomerular locations along the $\mathrm{D}-\mathrm{V}$ axis of the ventral $\mathrm{OB}$ is determined by anatomical locations of OSNs in the Vzone $[12,14]$.

The topographic projection along the $\mathrm{D}-\mathrm{V}$ axis of the $\mathrm{OB}$ is maintained by axon-axon and axon-target interactions. Several axon guidance molecules have been proposed to be involved in OSN axonal projections along the $\mathrm{D}-\mathrm{V}$ axis $[17-20]$. We have previously demonstrated that Neuropilin-2 (Nrp2)/Sema3F repulsive interactions between OSN axons play important roles in preserving the topographic order along the $\mathrm{D}-\mathrm{V}$ axis of the $\mathrm{OB}$ [21]. Nrp2 and Sema3F show complementary, gradient expressions in the OE. More specifically, the expression of Sema3F is high in the D-zone and low in the V-zone, while Nrp2 shows the opposite gradient. We also showed through gain and loss of function experiments that these molecules are necessary for the dorsal-ventral topographic projection in the OB. Further, we found a temporal difference in axonal extensions [21], whereby OSN axons project to the OB from in sequential dorsal to ventral order. These sequential projections are quite important to maintain the topographic order of the olfactory map because early-arriving D-zone OSN axons guide late-arriving V-zone OSN axons by secreting Sema3F. However, it is not clear how the differential timing of these axonal projections is regulated. We hypothesized that the timing of OSN production would be different depending on the location of OSNs in the OE. To test this, we examined spatiotemporal patterns of OSN neurogenesis during olfactory development.

\section{Materials and methods}

\section{Animals}

All experimental procedures were performed with the approval of the Animal Experiment Ethics Committee at the
University of Tokyo and according to the University of Tokyo guidelines for the care and use of laboratory animals.

\section{EdU and BrdU injections}

5-Ethynyl-2-deooxyuridine (EdU; Thermo Fisher Scientific) or 5-Bromo-2'-deoxyuridine (BrdU; Sigma-Aldrich) was intraperitoneally injected into mice at embryonic days $11.5,12.5,13.5,15.5$, and 17.5 , and postnatal days 0 and 2 $(50 \mathrm{mg} / \mathrm{kg})$.

\section{In situ hybridization and immunostaining}

In situ hybridization was performed according to previously described methods [21]. Primer sets used to generate RNA probes are listed in Additional file 1: Table S1.

Immunostaining was performed according to previously described methods [22]. Primary antibodies used are as follows: mouse anti-BrdU antibodies (1:500, Sigma-Aldrich); rabbit anti-OMACS antibodies (1:500); goat anti-OCAM antibodies (1:500, R\&D systems). Anti-OMACS antibodies were generated by immunizing rabbits with KLHconjugated synthetic peptides corresponding to 7-29 amino acid residue of the OMACS gene (operon biotechnologies). EdU signals were detected with the Click-iT EdU imaging kit (Thermostat Fisher Scientifics). To detect BrdU signal, sections were pre-treated for $1 \mathrm{~h}$ in $1.2 \mathrm{~N} \mathrm{HCl}$ at $37^{\circ} \mathrm{C}$ and then rinsed with $0.1 \mathrm{M}$ borate buffer ( $\mathrm{pH} 8.5$ ). After washout with phosphate-buffered saline (PBS), sections were incubated in a detection solution for $30 \mathrm{~min}$. Slides were then washed three times in PBS for 5 min and immunostained with anti-BrdU antibodies.

\section{Image acquisition and statistical analyses}

Optical and fluorescent images were photographed with a BZ-X700 microscope (Keyence). Images of three coronal $\mathrm{OE}$ slices were acquired and the number of $\mathrm{Br}(\mathrm{E}) \mathrm{dU}$-positive OSNs located within the NCAM-positive OE layer was manually counted. To define the OR-zones, serial OE sections at a thickness of $10 \mu \mathrm{m}$ were used. Each OE section was subjected to in situ hybridization using one of four OR probes (M72, P2, I7 and MOR28). After taking images, expression patterns of the OR genes were compared to determine the boundaries between the OR-zones. Percentages of labeled OSNs within the OR-zones were calculated as the number of labeled OSNs within each OR-zone divided by that in the entire OE region. All statistical analyses were performed with Origin Software (OriginLab).

\section{Results}

The D- and V-zones of the OE could be clearly separated on the basis of the expression of olfactory-specific medium chain acyl-CoA synthetase (OMACS) and olfactory-specific cell adhesion molecule (OCAM) [23-25], as OMACS is expressed specifically in the D-zone and OCAM in the V- 
zone (Fig. 1a and Additional file 2: Figure S1). Double staining of an OE section with antibodies against OMACS and OCAM delineated clear borders between the D- and Vzones of the OE (Fig. 1a and Additional file 2: Figure S1). To determine the timing of OSN production, we used either BrdU or EdU, which are incorporated into cells during the S-phase of the cell-cycle. EdU and BrdU were injected into pregnant mice at embryonic day 12.5 (E12.5) and E15.5, respectively. Then, distribution patterns of $\mathrm{Br}(\mathrm{E}) \mathrm{dU}$ - positive cells in the $\mathrm{OE}$ were analyzed at postnatal day 0 (P0). Using this approach, we observed that $\mathrm{Br}(\mathrm{E}) \mathrm{dU}$-positive OSNs were not uniformly distributed across the OE. EdU, which was used to label OSNs generated at E12.5, was predominantly detected in the D-zone of the $\mathrm{OE}$, and only lightly visible in the V-zone of the OE. On the other hand, a large number of E15.5-generated OSNs were located in the $\mathrm{V}$-zone of the $\mathrm{OE}$, while only a few were visible in the D-zone of the OE (Fig. 1b, c and d). The differential a
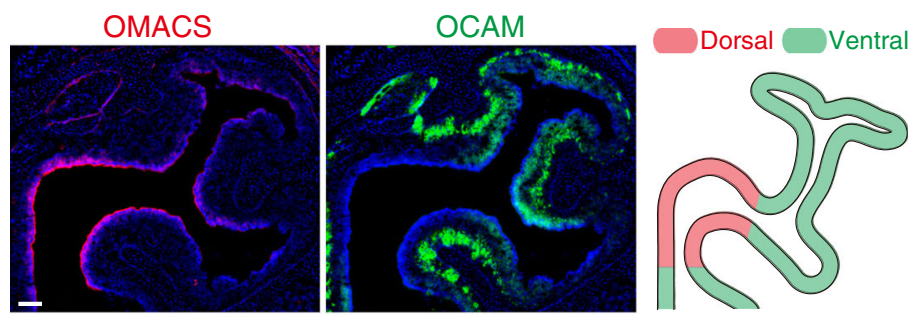

b
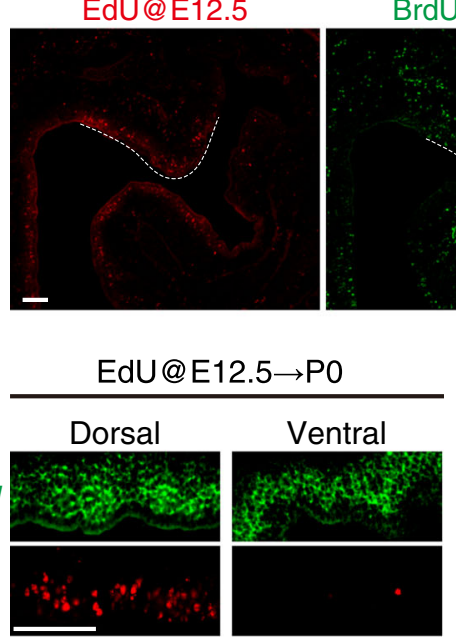

BrdU@E15.5

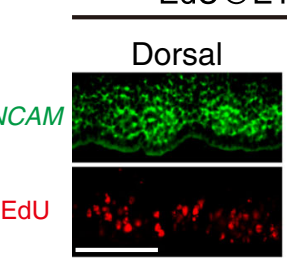

$\mathrm{EdU} @ \mathrm{E} 12.5 \rightarrow \mathrm{P0}$
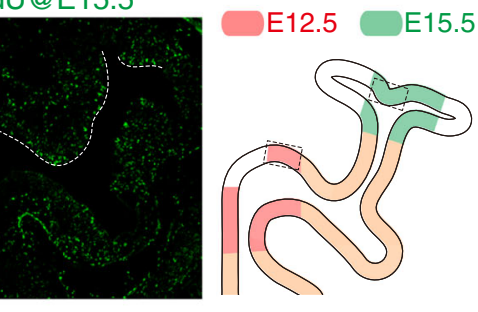

\section{C}

d

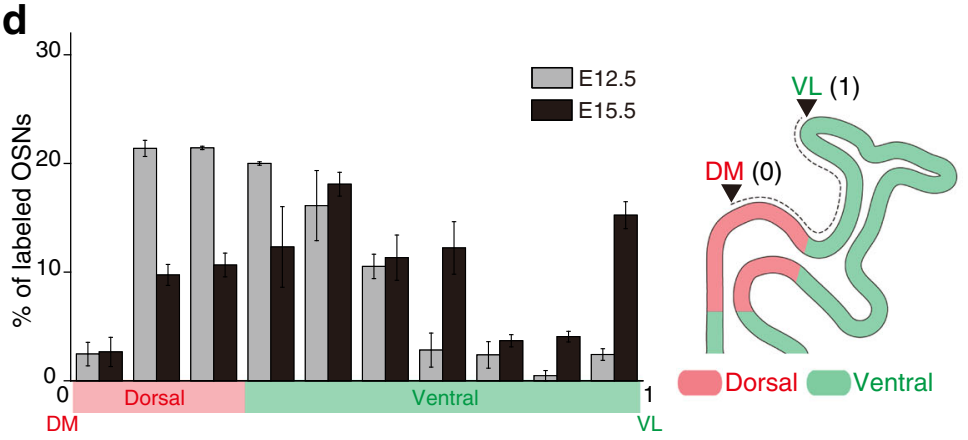

Fig. 1 Differential distribution patterns of E12.5- and E15.5-generated OSNs in the OE. a A coronal OE section from a postnatal day 0 (PO) mouse, immunostained with antibodies against OMACS (red) and OCAM (green). The OE section was counterstained with DAPI (blue). OMACS and OCAM are markers for the D- and V-zones, respectively. Schematic diagram of an OE section (shown on the right). b Distribution patterns of E12.5- and E15.5-generated OSNs in the OE. EdU and BrdU were injected at E12.5 and E15.5, respectively. OE sections from P0 mice were subjected to immunostaining with anti-BrdU antibodies and the Click reaction to detect EdU signals. $\mathbf{c}$ Enlarged images of the D- and V-zones of the OE. NCAM, an OSN marker, was detected by fluorescent in situ hybridization. d Percentages of OSNs generated at E12.5 and E15.5 were quantified in the OE. The OE sections were divided into ten subregions along with D-V axis. Percentages of labeled OSNs within the subregions were calculated as the number of labeled OSNs within each subregion divided by that in the entire OE region. Schematic diagram of the OE is shown on the right. $n=3$ slices from 2 to 3 animals, error bars indicate SEM. Scale bars, $100 \mu \mathrm{m}$ 
distribution patterns of $\mathrm{Br}(\mathrm{E}) \mathrm{dU}$-labeled OSNs suggest that the dynamics of OSN proliferation change during the course of development.

Encouraged by this result, we further examined the relationship between the timing of neurogenesis and OSN localization within the OE. We injected EdU at various developmental time points (E11.5, E13.5, E15.5, E17.5, and $\mathrm{P} 0$ ) and compared distribution patterns of EdUlabeled OSNs in the OE. The zone index [14] is typically used to quantify the location of OSNs. However, we were unable to apply the zone index to our study as it can only be used on OE sections from 3-week-old mice. Thus, we used another metric to quantify OSN location. We subdivided the OE region into four zones based on the expression patterns of the following four wellcharacterized OR genes: M72, P2, I7, and MOR28. Each of the selected genes showed unique and nonoverlapping expression patterns in the OE at P7 (Fig. 2a). We then calculated the proportion of EdU-labeled cells in each zone. As a result, we found that the percentage of E13.5-generated OSNs was much higher in the P2-zone than in the MOR28-zone. In contrast, the percentage of
P0-generated OSNs was higher in the MOR28-zone than in the P2-zone (Fig. 2b and c). Since the P2-zone occupies the more dorsomedial region of the OE than the MOR28zone, these results suggest that OSNs are gradually produced from the dorsomedial (DM) to the ventrolateral (VL) direction within the ventral OE. However, no positional differences were observed in the distribution patterns of EdU-positive OSNs within the M72-zone, which corresponds to the D-zone. In the D-zone, the largest number of EdU-positive OSNs was produced around E17.5 (Fig. 2c). It should be mentioned that no obvious EdU-positive OSNs were visible in E11.5 EdU-injected mice (data not shown). It could be that the incorporation of EdU by OSN precursor cells was diluted due to cell division, to the degree that the EdU signal was reduced to background levels.

Nrp2 expression can be used as another index of OSN location, as Nrp2 shows a VL-high to DM-low expression gradient in the OE (Fig. 3a). Further, the expression level of Nrp2 determines glomerular locations along the $\mathrm{D}-\mathrm{V}$ axis of the $\mathrm{OB}$ [21]. We then compared distribution patterns of EdU-labeled OSNs and Nrp2 expression a
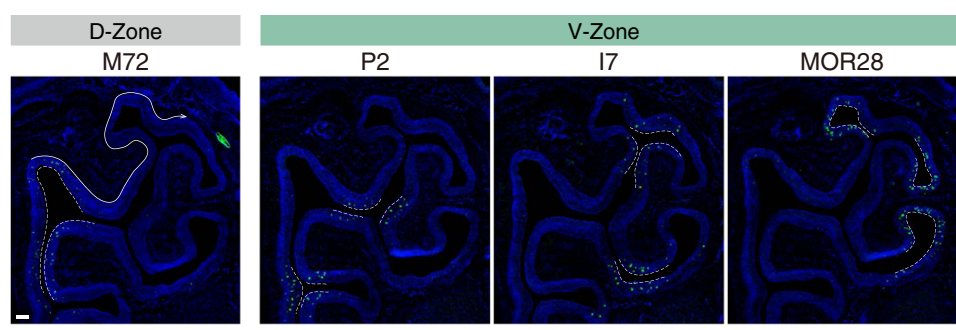

b

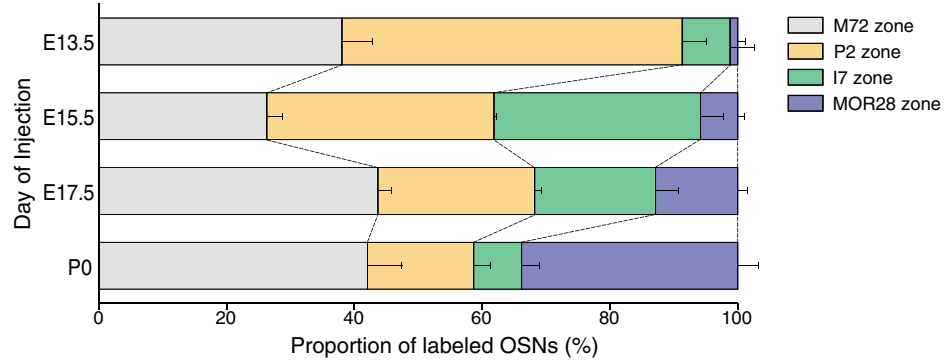

C

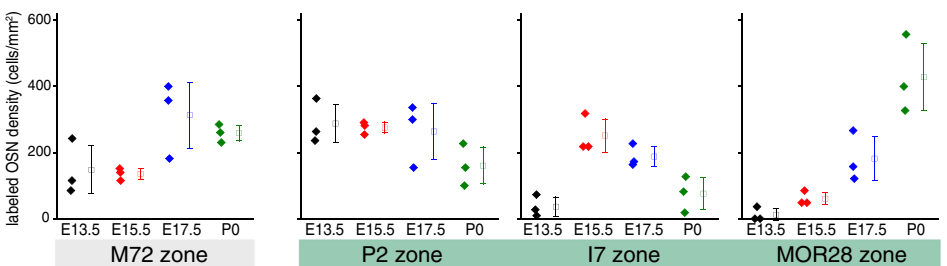

Fig. 2 Spatial- and temporal-specific patterns of OSN neurogenesis in the OE. a In situ hybridization of OE sections from a P7 mouse using probes for OR genes (M72, P2, 17, and MOR28). The OE was subdivided into four zones based on OR gene expression patterns. The zones defined by the ORs are indicated by dashed lines. Note that the M72-area corresponds to the D-zone of the OE. b Proportions of EdU-labeled OSNs within the ORdefined zones. EdU injection was performed at E13.5, 15.5, 17.5, and PO, and distribution patterns were analyzed at P7. c Quantification of proliferation levels, defined as the density of EdU-labeled OSNs within OR-defined zones. $n=3$ sections from 3 animals, error bars indicate SEM. Scale bar, $100 \mu m$ 
a

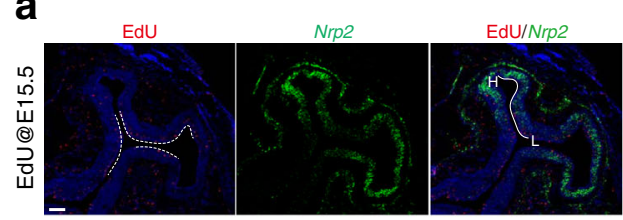

EdU

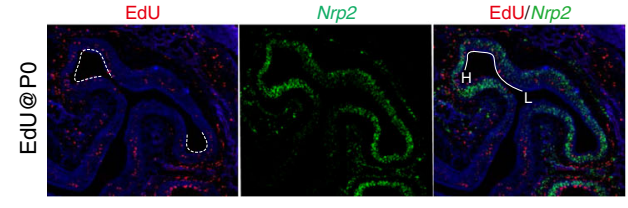

C

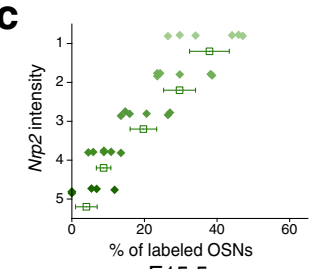

E15.5

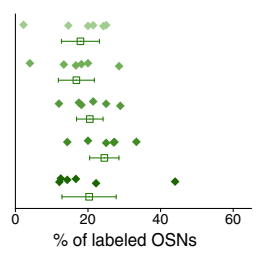

E17.5

b

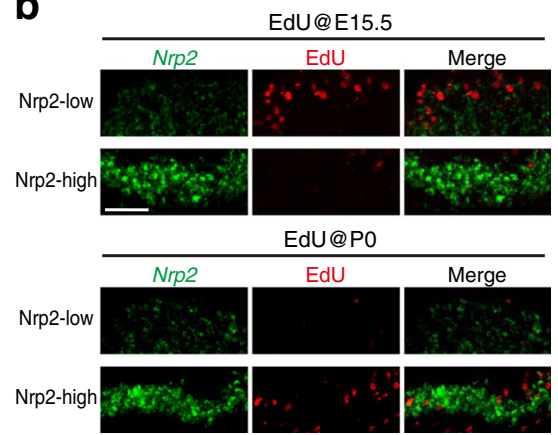

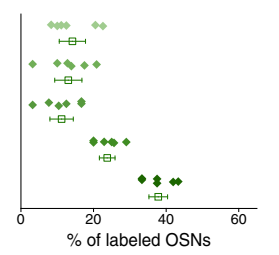

PO

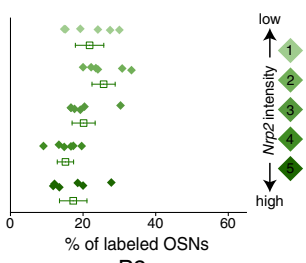

P2

Fig. 3 Sequential proliferation of OSNs from DM (Nrp2-low) to VL (Nrp2-high) within the V-zone of the OE. a Distribution patterns of E15.5- and PO-generated OSNs in the OE. EdU injection was performed at E15.5 and PO. After the injections, coronal OE sections from P7 mice were subjected to in situ hybridization using probes for Nrp2. EdU signals were detected with the Click reaction. EdU-positive OSNs were located in the area indicated by the dashed lines. Nrp2 can be visualized as being expressed in a DM-low to VL-high gradient. H Nrp2-high, L Nrp2-low. $\mathbf{b}$ Enlarged images of Nrp2-high $(\mathrm{VL})$ and Nrp2-low (DL) zones. c The kinetics of OSN proliferation were spatially different within the V-zone of the OE. The ventral OE was divided into five subzones according to Nrp2 expression levels (right). The level of proliferation in each subzone was quantified as the percentage of EdU-positive cells. $n=6$ areas from 3 animals, error bars indicate SEM. Scale bars, $100 \mu \mathrm{m}$

(Fig. 3a and b). Using this approach, it was evident that a greater number of E15.5-generated OSNs were located in the Nrp2-low area than in the Nrp2-high area. However, the percentage of P0-generated OSNs was much higher in the Nrp2-high area than in the Nrp2-low area. The percentage of E17.5-generated OSNs was comparable between the two regions (Fig. 3b and c). Together, these results indicate that the area with the most EdUlabeled OSNs shifted from DM to VL within the V-zone of the $\mathrm{OE}$ during embryonic development. It should be noted that P2-generated OSNs were uniformly distributed (Fig. 3c). Given that Nrp2-low OSN axons projected to the OB earlier than Nrp2-high OSN axons, it is possible that the timing of OSN axonal projection is determined by that of OSN neurogenesis.

\section{Discussion}

The development of neural circuits relies on the timely production of neurons. In this study, we examined spatiotemporal patterns of OSN neurogenesis during olfactory development. In the mouse olfactory system, the positional information of OSNs in the OE regulates the expression of OR genes and axon guidance molecules, thereby correlating OR identity with glomerular locations along the $\mathrm{D}-\mathrm{V}$ axis. In this study, we provide the first evidence that OSN positional information also controls the timing of OSN proliferation. Support of this was provided by the observation that OSN production rate was not uniform across the OE. Moreover, we found that the area with the most $\mathrm{Br}(\mathrm{E}) \mathrm{dU}$-labeled OSNs shifted from $\mathrm{DM}$ to VL during the course of development, suggesting that cell proliferation propagates in a DM-VL direction across the OE (Fig. 4).

What is the significance of the differential timing of OSN neurogenesis? The timing of neurogenesis is a determinant for cell-fate specification and precise neural circuitry [26, 27]. For example, it has been reported that the timing of neurogenesis determines the location of secondary olfactory mitral cells as well as their axonal projections [28]. However, in the primary olfactory system, region-specific OR gene expression is already observed at E13.5 [29, 30]. Furthermore, other regional markers, such as Nrp2, OMACS, and OCAM, are expressed as early as E12.5 [21, 24]. These observations indicate that the fate of OSNs is already specified at the early embryonic stage. Thus, it is unlikely that the timing of neurogenesis determines the expression of ORs nor does it determine the projection site of OSN axons.

OSN axons project to the $\mathrm{OB}$ in a dorsal to ventral direction. The sequential projections of OSN axons are known to be involved in formation of the olfactory map along the $\mathrm{D}-\mathrm{V}$ axis [21]. Our present study 


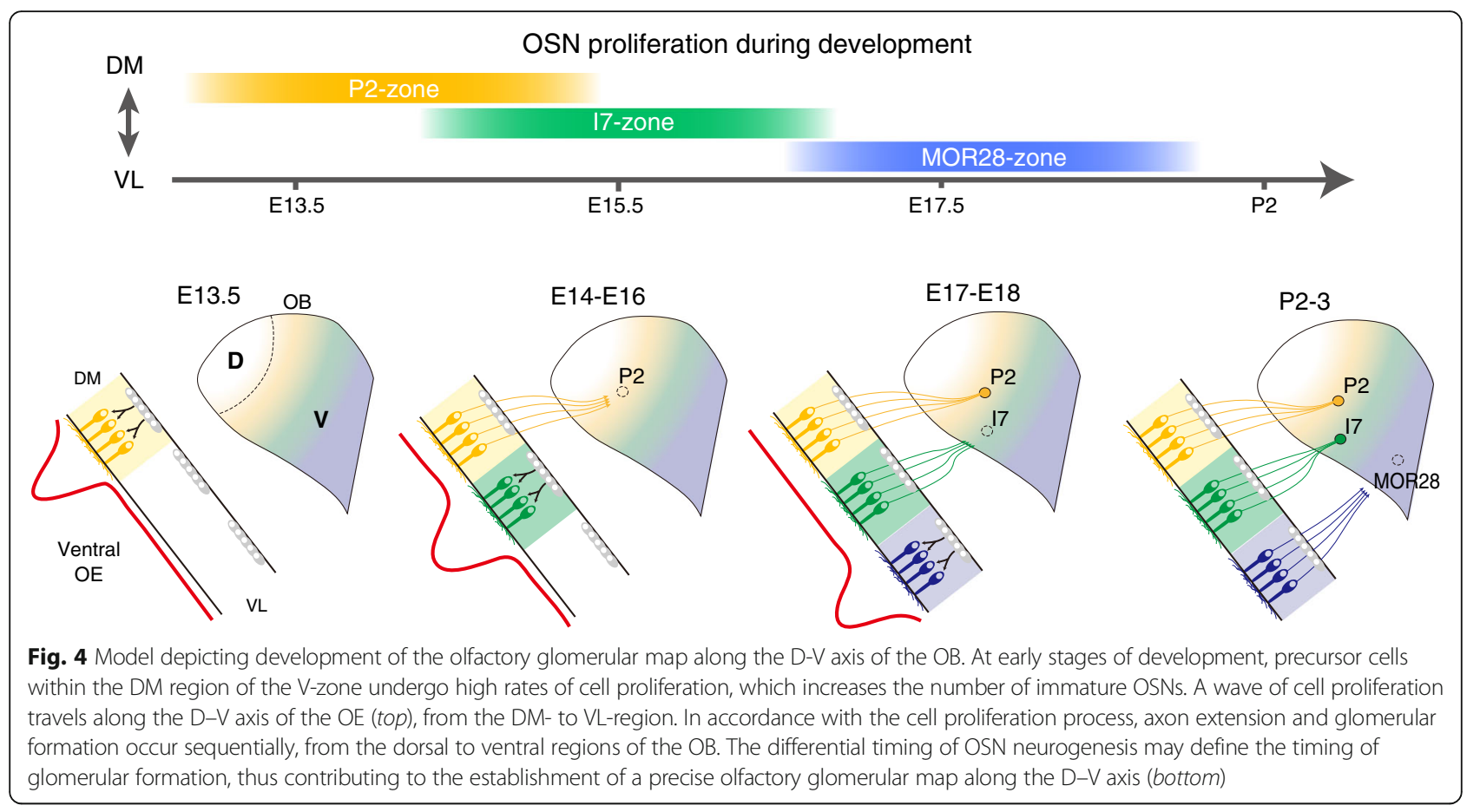

indicated that the sequence of OSN axonal projections correlated with that of OSN proliferation in the $\mathrm{V}$-zone of the OE. This result prompted us to think that the timing of OSN neurogenesis contributed to olfactory map formation through the regulation of axonal projection timing (Fig. 4). It has been reported that glomerular structures first emerge from the anterodorsal region of the $\mathrm{OB}$ [31]. Moreover, several experiments using fluorescent protein-tagged OSN axons have revealed that glomeruli for P2, I7, and MOR28 first emerge around E17.5-18.5, P0-1, and P3-5, respectively (Table 1 and Fig. 4) [5, 32-35]. These glomeruli are aligned in the order of P2, I7, and MOR28 along the $\mathrm{D}-\mathrm{V}$ axis of the OB. Given that a certain number of OSN axons are required to form a glomerular structure, these observations point toward an intriguing possibility that the spatial- and temporal-specific proliferation of OSNs could regulate the timing of glomerular formation by controlling the number of OSN axons at a particular target.
In contrast to the V-zone of the $\mathrm{OE}$, we were unable to find a positional difference in the rate of OSN production within the D-zone of the OE (Fig. 2b). It could be that different mechanisms are responsible for mediating OSN neurogenesis in the D-zone of the OE. In this study, we observed a burst of proliferation twice in the D-zone: the first one was at E12.5 and the second one was at E17.5 (Figs. 1d and 2c). D-zone ORs are classified into two phylogenetically different groups; Class I and Class II [36]. These two groups constitute distinct glomerular domains in the dorsal $\mathrm{OB}$; the anterior region comprises Class I-ORs while the posterior region comprises Class II-ORs [13, 37]. We assumed that the first burst of proliferation reflects the proliferation for Class I OR-expressing OSNs, and the second one reflects that for Class II OR-expressing OSNs. Comparing the difference in the timing of neurogenesis between Class I ORand Class II OR-expressing OSNs may give us some insight into the mechanisms underlying the formation of these domains in the OB.

Table 1 The timing of OSN proliferation in the OE and glomerular formation in the OB

\begin{tabular}{|c|c|c|c|}
\hline \multicolumn{2}{|l|}{ OE } & \multicolumn{2}{|l|}{$\mathrm{OB}$} \\
\hline OE region & $\begin{array}{l}\text { Timing of most active } \\
\text { OSN proliferation }\end{array}$ & OR type & $\begin{array}{l}\text { Timing of glomerular } \\
\text { formation }\end{array}$ \\
\hline M72-zone (D) & ND & M72 & PD2-3 [35] \\
\hline P2-zone (V) & E13.5 & P2 & E17.5-18.5 [5, 34] \\
\hline I7-zone (V) & E15.5 & 17 & PD0-1 [32] \\
\hline MOR28-zone (V) & PO & MOR28 & PD3-5 [33] \\
\hline
\end{tabular}




\section{Additional files}

Additional file 1: Table S1. PCR primer sets used to generate RNA probes for in situ hybridization. (DOCX $13 \mathrm{~kb}$ )

Additional file 2: Figure S1. Separation of the D- and V-zones of the $\mathrm{OE}$ on the basis of markers. (A) Immunostaining of an OE section with antibodies directed against OMACS and OCAM. OMACS and OCAM are markers for the D- and V-zones, respectively. Enlarged photos are shown on the bottom. White dotted lines show the boundaries of the D- and $V_{-}$ zones. The OE section was counterstained with DAPI. (B) Immunostaining of an OE section with antibodies directed against OMACS and NQO1. Both molecules were co-expressed in the D-zone of the OE. Note that OMCAS was expressed not only in the OE but also in the lamina propria. Enlarged photos are shown on the bottom. White dotted lines show the boundaries of the D- and V-zones. The OE section was counterstained with DAPI. (PDF 2969 kb)

\section{Abbreviations}

OB: Olfactory bulb; OCAM: Olfactory-specific cell adhesion molecule; OE: Olfactory epithelium; OMACS: Olfactory-specific medium acyl-CoA synthetase (OMACS); OR: Olfactory receptor; OSN: Olfactory sensory neuron

\section{Acknowledgements}

We thank A. Nakashima for her help in preparing the manuscript

\section{Funding}

This work was supported by the Takeda Science Foundation, JST-PREST and JSPS KAKENHI Grant Number, 16H06144.

\section{Availability of data and materials}

The datasets generated and analysed during the current study are available in the figshare repository (https://doi.org/10.6084/m9.figshare.4580509.v1).

\section{Authors' contributions}

$\mathrm{E}, \mathrm{NI}$ and HT conceived the experiments. E, NI, YI and HT wrote the manuscript. $\mathrm{E}, \mathrm{NI}$ and $\mathrm{BL}$ performed the experiments and analyzed the data. All authors contributed to the writing and provided helpful comments. All authors read and approved the final manuscript.

\section{Competing interests}

The authors declare that they have no competing interests.

\section{Consent for publication}

Not applicable.

\section{Ethics approval and consent to participate}

Not applicable.

\section{Author details}

${ }^{1}$ Division of Innate Immunity, Department of Microbiology and Immunology, the Institute of Medical Science, the University of Tokyo, Tokyo 108-8639, Japan. ${ }^{2}$ Laboratory of Chemical Pharmacology, Graduate School of Pharmaceutical Sciences, the University of Tokyo, Tokyo 113-0033, Japan. ${ }^{3}$ Center for Information and Neural Networks, National Institute of Information and Communications Technology, Suita City, Osaka 565-0871, Japan. ${ }^{4}$ Japan Science and Technology Agency (JST), PRESTO, 4-1-8 Honcho Kawaguchi, Saitama 332-0012, Japan.

Received: 13 December 2016 Accepted: 6 February 2017 Published online: 13 February 2017

\section{References}

1. Buck $L$, Axel R. A novel multigene family may encode odorant receptors: a molecular basis for odor recognition. Cell. 1991;65(1):175-87.

2. Serizawa S, Miyamichi K, Nakatani H, Suzuki M, Saito M, Yoshihara Y, Sakano $H$. Negative feedback regulation ensures the one receptor-one olfactory neuron rule in mouse. Science. 2003;302(5653):2088-94.

3. Ressler KJ, Sullivan SL, Buck LB. Information coding in the olfactory system: evidence for a stereotyped and highly organized epitope map in the olfactory bulb. Cell. 1994;79(7):1245-55.
4. Vassar R, Chao SK, Sitcheran R, Nunez JM, Vosshall LB, Axel R. Topographic organization of sensory projections to the olfactory bulb. Cell. 1994;79(6): 981-91.

5. Mombaerts P, Wang F, Dulac C, Chao SK, Nemes A, Mendelsohn M, Edmondson J, Axel R. Visualizing an olfactory sensory map. Cell. 1996;87(4): 675-86.

6. Takeuchi H, Sakano H. Neural map formation in the mouse olfactory system. Cell Mol Life Sci. 2014;71(16):3049-57.

7. Mori K, Sakano H. How is the olfactory map formed and interpreted in the mammalian brain? Annu Rev Neurosci. 2011;34:467-99.

8. Wang F, Nemes A, Mendelsohn M, Axel R. Odorant receptors govern the formation of a precise topographic map. Cell. 1998;93(1):47-60.

9. Imai T, Suzuki M, Sakano H. Odorant receptor-derived CAMP signals direct axonal targeting. Science. 2006;314(5799):657-61.

10. Nakashima A, Takeuchi H, Imai T, Saito H, Kiyonari H, Abe T, Chen M, Weinstein LS, Yu CR, Storm DR, et al. Agonist-independent GPCR activity regulates anterior-posterior targeting of olfactory sensory neurons. Cell. 2013;154(6):1314-25

11. Col JA, Matsuo T, Storm DR, Rodriguez I. Adenylyl cyclase-dependent axonal targeting in the olfactory system. Development. 2007;134(13):2481-9.

12. Astic L, Saucier D, Holley A. Topographical relationships between olfactory receptor cells and glomerular foci in the rat olfactory bulb. Brain Res. 1987; 424(1):144-52.

13. Tsuboi A, Miyazaki T, Imai T, Sakano H. Olfactory sensory neurons expressing class I odorant receptors converge their axons on an antero-dorsal domain of the olfactory bulb in the mouse. Eur J Neurosci. 2006;23(6):1436-44.

14. Miyamichi K, Serizawa S, Kimura HM, Sakano H. Continuous and overlapping expression domains of odorant receptor genes in the olfactory epithelium determine the dorsal/ventral positioning of glomeruli in the olfactory bulb. J Neurosci Off J Soc Neurosci. 2005;25(14):3586-92.

15. Vassar R, Ngai J, Axel R. Spatial segregation of odorant receptor expression in the mammalian olfactory epithelium. Cell. 1993;74(2):309-18.

16. Ressler KJ, Sullivan SL, Buck LB. A zonal organization of odorant receptor gene expression in the olfactory epithelium. Cell. 1993;73(3):597-609.

17. Norlin EM, Alenius M, Gussing F, Hagglund M, Vedin V, Bohm S. Evidence for gradients of gene expression correlating with zonal topography of the olfactory sensory map. Mol Cell Neurosci. 2001;18(3):283-95.

18. Nguyen-Ba-Charvet KT, Di Meglio T, Fouquet C, Chedotal A. Robos and slits control the pathfinding and targeting of mouse olfactory sensory axons. J Neurosci Off J Soc Neurosci. 2008:28(16):4244-9.

19. Cho JH, Lepine M, Andrews W, Parnavelas J, Cloutier JF. Requirement for Slit-1 and Robo-2 in zonal segregation of olfactory sensory neuron axons in the main olfactory bulb. J Neurosci Off J Soc Neurosci. 2007; 27(34):9094-104.

20. Walz A, Rodriguez I, Mombaerts P. Aberrant sensory innervation of the olfactory bulb in neuropilin-2 mutant mice. J Neurosci Off J Soc Neurosci. 2002;22(10):4025-35.

21. Takeuchi H, Inokuchi K, Aoki M, Suto F, Tsuboi A, Matsuda I, Suzuki M, Aiba A, Serizawa S, Yoshihara Y, et al. Sequential arrival and graded secretion of Sema3F by olfactory neuron axons specify map topography at the bulb. Cell. 2010;141(6):1056-67.

22. Serizawa S, Miyamichi K, Takeuchi H, Yamagishi Y, Suzuki M, Sakano H. A neuronal identity code for the odorant receptor-specific and activitydependent axon sorting. Cell. 2006;127(5):1057-69.

23. Gussing F, Bohm S. NQO1 activity in the main and the accessory olfactory systems correlates with the zonal topography of projection maps. Eur J Neurosci. 2004;19(9):2511-8.

24. Oka Y, Kobayakawa K, Nishizumi H, Miyamichi K, Hirose S, Tsuboi A, Sakano H. O-MACS, a novel member of the medium-chain acyl-CoA synthetase family, specifically expressed in the olfactory epithelium in a zone-specific manner. Eur J Biochem. 2003;270(9):1995-2004.

25. Yoshihara Y, Kawasaki M, Tamada A, Fujita H, Hayashi H, Kagamiyama H, Mori K. OCAM: a new member of the neural cell adhesion molecule family related to zone-to-zone projection of olfactory and vomeronasal axons. J Neurosci Off J Soc Neurosci. 1997;17(15):5830-42.

26. Molyneaux BJ, Arlotta P, Menezes JR, Macklis JD. Neuronal subtype specification in the cerebral cortex. Nat Rev Neurosci. 2007;8(6):427-37.

27. Rakic $\mathrm{P}$, Ayoub $\mathrm{AE}$, Breunig JJ, Dominguez $\mathrm{MH}$. Decision by division: making cortical maps. Trends Neurosci. 2009;32(5):291-301.

28. Imamura F, Ayoub AE, Rakic $P$, Greer CA. Timing of neurogenesis is a determinant of olfactory circuitry. Nat Neurosci. 2011;14(3):331-7. 
29. Sullivan SL, Bohm S, Ressler KJ, Horowitz LF, Buck LB. Target-independent pattern specification in the olfactory epithelium. Neuron. 1995;15(4):779-89.

30. Rodriguez-Gil DJ, Treloar HB, Zhang X, Miller AM, Two A, Iwema C, Firestein SJ, Greer CA. Chromosomal location-dependent nonstochastic onset of odor receptor expression. J Neurosci Off J Soc Neurosci. 2010;30(30):10067-75.

31. Bailey MS, Puche AC, Shipley MT. Development of the olfactory bulb: evidence for glia-neuron interactions in glomerular formation. J Comp Neurol. 1999;415(4):423-48.

32. Valle-Leija P, Blanco-Hernandez E, Drucker-Colin R, Gutierrez-Ospina G, Vidaltamayo R. Supernumerary formation of olfactory glomeruli induced by chronic odorant exposure: a constructivist expression of neural plasticity. PLoS One. 2012;7(4):e35358.

33. Sengoku S, Ishii T, Serizawa S, Nakatani H, Nagawa F, Tsuboi A, Sakano H. Axonal projection of olfactory sensory neurons during the developmental and regeneration processes. Neuroreport. 2001;12(5):1061-6.

34. Royal SJ, Key B. Development of P2 olfactory glomeruli in P2-internal ribosome entry site-tau-LacZ transgenic mice. J Neurosci Off J Soc Neurosci. 1999;19(22):9856-64

35. Potter SM, Zheng C, Koos DS, Feinstein P, Fraser SE, Mombaerts P. Structure and emergence of specific olfactory glomeruli in the mouse. J Neurosci Off J Soc Neurosci. 2001;21(24):9713-23.

36. Zhang $X$, Firestein $\mathrm{S}$. The olfactory receptor gene superfamily of the mouse. Nat Neurosci. 2002;5(2):124-33.

37. Bozza T, Vassalli A, Fuss S, Zhang JJ, Weiland B, Pacifico R, Feinstein P, Mombaerts P. Mapping of class I and class II odorant receptors to glomerular domains by two distinct types of olfactory sensory neurons in the mouse. Neuron. 2009;61(2):220-33.

\section{Submit your next manuscript to BioMed Central and we will help you at every step:}

- We accept pre-submission inquiries

- Our selector tool helps you to find the most relevant journal

- We provide round the clock customer support

- Convenient online submission

- Thorough peer review

- Inclusion in PubMed and all major indexing services

- Maximum visibility for your research

Submit your manuscript at www.biomedcentral.com/submit

C) Biomed Central 